\title{
Audiovisual Processing is Abnormal in Parkinson's Disease and Correlates with Freezing of Gait and Disease Duration
}

\author{
Conor Fearon \\ Dublin Neurological Institute at the Mater Misericordiae University Hospital \\ John Butler \\ Technological University Dublin, john.s.butler@tudublin.ie \\ Louise Newman \\ Trinity College Dublin, Ireland
}

See next page for additional authors

Follow this and additional works at: https://arrow.tudublin.ie/scschmatart

Part of the Mathematics Commons

\section{Recommended Citation}

Fearon, C. et al. (2015). Audiovisual Processing is Abnormal in Parkinson's Disease and Correlates with Freezing of Gait and Disease Duration. Journal of Parkinson's Disease Oct 17;5(4):925-36. doi: 10.3233/ JPD-150655.

This Article is brought to you for free and open access by the School of Mathematics at ARROW@TU Dublin. It has been accepted for inclusion in Articles by an authorized administrator of ARROW@TU Dublin. For more information, please contact arrow.admin@tudublin.ie, aisling.coyne@tudublin.ie,gerard.connolly@tudublin.ie.

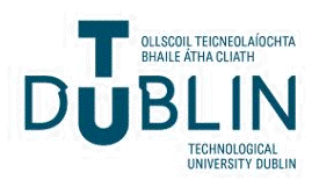




\section{Authors}

Conor Fearon, John Butler, Louise Newman, Timothy Lynch, and Richard B. Reilly

This article is available at ARROW@TU Dublin: https://arrow.tudublin.ie/scschmatart/202 


\title{
Audiovisual Processing is Abnormal in Parkinson's Disease and Correlates with Freezing of Gait and Disease Duration
}

\author{
Conor Fearon ${ }^{\mathrm{a}, \mathrm{b}, 1}$, John S. Butler ${ }^{\mathrm{b}, 1}$, Louise Newman ${ }^{\mathrm{a}, \mathrm{b}}$, Timothy Lynch ${ }^{\mathrm{a}, *}$ and Richard B. Reilly ${ }^{\mathrm{b}, \mathrm{c}, *}$ \\ ${ }^{\mathrm{a}}$ Dublin Neurological Institute at the Mater Misericordiae University Hospital, Dublin 7, Ireland \\ ${ }^{\mathrm{b}}$ Trinity Centre for Bioengineering, Trinity College Dublin, Dublin 2, Ireland \\ ${ }^{\mathrm{c}}$ School of Medicine, Trinity College Dublin, Dublin 2, Ireland
}

\begin{abstract}
.
Background: Sensory and perceptual disturbances progress with disease duration in Parkinson's disease (PD) and probably contribute to motor deficits such as bradykinesia and gait disturbances, including freezing of gait (FOG). Simple reaction time tests are ideal to explore sensory processing, as they require little cognitive processing. Multisensory integration is the ability of the brain to integrate sensory information from multiple modalities into a single coherent percept, which is crucial for complex motor tasks such as gait.

Objectives: The aims of this study were to: 1 . Assess differences in unisensory (auditory and visual) and multisensory processing speed in people with PD and age-matched healthy controls. 2. Compare relative differences in unisensory processing in people with PD with disease duration and freezing of gait status taking into account the motor delays, which are invariably present in PD. 3. Compare relative differences in multisensory (audiovisual) processing between the PD cohort and age-matched controls. Methods: 39 people with PD (23 with FOG) and 17 age-matched healthy controls performed a reaction time task in response to unisensory (auditory-alone, visual-alone) and multisensory (audiovisual) stimuli.

Results: The PD group were significantly slower than controls for all conditions compared with healthy controls but auditory reaction times were significantly faster than visual for the PD group only. These relative unisensory differences are correlated with disease duration and divide the PD group by FOG status, but these factors are co-dependent. Although multisensory facilitation occurs in PD, it is significantly less enhanced than in healthy controls.

Conclusion: There are significant unisensory and multisensory processing abnormalities in PD. The relative differences in unisensory processing are specific to PD progression, providing a link between these sensory abnormalities and a motor feature of PD. Sensory disturbances have previously been postulated to be central to FOG but this is the first study to predict audiovisual processing abnormalities using FOG status. The multisensory processing abnormalities are independent of disease duration and FOG status and may be a potential biomarker for the disease.
\end{abstract}

Keywords: Parkinson's disease, sensory processing, multisensory, auditory, visual

\section{INTRODUCTION}

Sensory and perceptual disturbances are common ${ }^{1}$ C. Fearon and J.S. Butler contributed equally to the paper.

* Correspondence to: Timothy Lynch, Dublin Neurological Institute at the Mater Misericordiae University Hospital, 57 Eccles Street, Dublin 7, Ireland. E-mail: tlynch@dni.ie and Richard B. Reilly, Trinity Centre for Bioengineering, The School of Medicine and the School of Engineering, Trinity College Dublin, Ireland. E-mail: rbreilly@tcd.ie. in Parkinson's disease (PD) [1-3]. Subtle deficits of the sensory system, often not detected by routine examination, occur in people with Parkinson's disease (PwP). From simple anosmia and impaired kinesthetic perception, to more complex visual hallucinations and spatiotemporal perceptual abnormalities, altered 
sensory processing is found across multiple modalities [4-8]. Of note, integration of multiple environmental sensory inputs is crucial for a refined but complex goal-directed motor output (e.g. locomotion through a crowded environment). There is increasing evidence that these sensory deficits contribute to the pathophysiology of some of the abnormal motor features of PD [9-11], including freezing of gait (FOG), where patients feel as though their feet are momentarily glued to the floor [12], and which is closely associated with falls and nursing home placement [13]. Although the underlying pathophysiology FOG is incompletely understood, sensory mechanisms are likely to be core factors underlying this motor symptom [14].

There are many studies quantifying single modality (unisensory) deficits in PD. Simple reaction times are helpful when exploring sensory responses, as they require little cognitive processing (interpretation can be difficult in a patient population where cognitive impairment is common). Simple reaction times to auditory and visual stimuli are delayed in $\mathrm{PwP}$ as compared to healthy controls [15-22]. However, motor output in response to sensory stimuli requires both sensory processing and sensorimotor integration. Simple unisensory reaction times are, therefore, delayed in PwP because of bradykinesia, and do not solely assess sensory differences in these patients, as the response is a combination of motor and sensory processing pathways. Quantitative assessment of sensory processing speeds therefore requires examination of relative differences in response times to stimuli, separate from common motor output time. Nevertheless, premotor delays in processing have been shown in PwP via movement-related potentials $[21,23]$ and auditory, visual and somatosensory evoked potentials [24-27], implying that unisensory processing is altered in PD, independent of motor integration.

Multisensory integration is the brain's ability to integrate sensory information from multiple modalities into a single coherent percept, leading to increased speed and accuracy of response [28]. When reaction times to multisensory stimuli are compared to individual component unisensory stimuli, the responses are significantly faster than would be predicted based on the unisensory reaction times. By comparing relative response times to unisensory and multisensory stimuli, quantitative assessment of multisensory integration can be performed, while controlling for variable motor response times in PD.

Multisensory integration is enhanced in healthy elderly populations [29] but it is unknown if this multisensory facilitation is present in PwP. Inefficient multisensory integration is linked with falls in older adults, highlighting the importance of controlled multisensory processing in balance and locomotor control [30]. Given that locomotion is highly multisensory task and that progressive gait impairment frequently occurs in $\mathrm{PD}$, abnormal multisensory processing may occur in PD. Single cell animal studies have highlighted the basal ganglia as an important multisensory hub $[31,32]$. As PD is a basal ganglia disorder and has widespread sensory abnormalities, we hypothesized that multisensory integration is altered in PD.

Few studies have reported multisensory abnormalities in PD [33]. The multisensory interactions between auditory and visual stimuli have not been studied in PD. We studied PwP and age-matched healthy controls performing a reaction time task in response to unisensory (auditory-alone, visual-alone) and multisensory (audiovisual) stimuli. In this study we have made efforts to limit the effect of attention by comparing relative differences between audio, visual and audiovisual response times. In this way, each participant acts as his or her own control. Thus any differences in performance represent relative differences in either processing of different modalities or shifts in modality-specific attention between groups. Given the widespread sensory abnormalities in PD, we hypothesized that multisensory integration is also altered in PwP. The reaction time task was used in order to:

1. Assess differences in unisensory (auditory and visual) processing speed in PwP and age-matched healthy controls.

2. Correlate relative differences in unisensory (auditory vs visual) processing in PwP with disease duration and FOG status taking into account the known motor delays in PD.

3. Compare relative differences in multisensory processing between $\mathrm{PwP}$ and age-matched controls.

\section{METHODS}

\section{Participants}

39 patients with idiopathic PD (as defined by the UK Brain Bank Criteria [34]; Modified Hoehn and Yahr stage II-IV) were recruited from the Movement Disorder Clinic at the Dublin Neurological Institute. Ethical approval was granted from the hospital ethics committee and informed consent was obtained from all participants. All patients underwent clinical and neuropsychological testing including Montreal 
Cognitive Assessment (MoCA), Frontal Assessment Battery (FAB) and Unified Parkinson's Disease Rating Scale III (UPDRS III). FOG status was recorded for all patients based on Question 1 of the New Freezing of Gait Questionnaire ("Did you experience a freezing episode over the past month?") [35]. All participants had normal corrected vision and hearing and were tested in the "on"-state. A group of 17 age-matched healthy controls were recruited among hospital staff and relatives of participants for comparison. The control group had no neurological comorbidities and normal cognition.

\section{Stimuli}

Participants performed a simple reaction time task consisting of three stimulus conditions: "auditory" (A), "visual" (V) and "audiovisual" (AV). Stimuli were presented using Presentation software (Neurobehavioral Systems, Inc., Albany CA). The auditory condition consisted of a 1000-Hz tone (duration 60 msecs; $75 \mathrm{~dB}$; rise/fall time 5 msecs), presented from via inbuilt speakers of a Dell laptop (Latitude E5530). The visual condition consisted of a red disc with a diameter of $3.2 \mathrm{~cm}$ (subtending 1.5 degrees in diameter at a viewing distance of $122 \mathrm{~cm}$ ) appearing on a black background, presented on the screen for 60 milliseconds. The audiovisual condition consisted of the auditory and visual conditions presented simultaneously.

\section{Procedure}

Participants were seated in front the laptop and instructed to press a button as quickly as possible when they saw the red circle, or heard the tone, or saw the circle and heard the tone together. The stimulus conditions were presented with equal probability and in random order in blocks of 100 trials. Inter-stimulusinterval (ISI) varied randomly between 1000 and 3000 milliseconds according to a uniform (square wave) distribution. Participants completed 3 blocks, resulting in 100 repetitions per stimulus condition. These methods are also presented in detail elsewhere [36-41]. The range of reaction times accepted was determined at the individual participant level with the slowest cut off at 150 milliseconds and fastest $2.5 \%$ of trials excluded.

\section{Statistical analysis}

Data were processed and analyzed using custom MATLAB (Mathworks, Natick, MA) scripts and SPSS 22.
Reaction time analysis

Mean reaction times for each condition were calculated for all participants. A mixed one-way analysis of variance (ANOVA), with the factors of stimulus condition (auditory-alone, visual-alone, audiovisual) and group ( $\mathrm{PwP}$ and control participants) was performed to compare the reaction times of the three stimulus conditions between PwP and controls. Post-hoc comparisons between the conditions were performed to test for the presence of relative differences between the unisensory conditions as a well as faster reaction times in the multisensory condition. In order to examine whether differences in capacity for focused attention differed between groups, reaction times and hit rates were calculated for the first and last blocks of trials in each group.

\section{Relative sensory processing and FOG status}

To investigate the relationship between relative sensory processing (controlling for motor delays) and FOG status, the PwP group was subdivided by Question 1 of the New Freezing of Gait Questionnaire, as described above [35]. A mixed repeated ANOVA was performed with the within-participant factor of relative reaction time (auditory-visual vs audiovisual-visual vs audiovisual-auditory) and between-participant factor of FOG status (freezers vs non-freezers). The reaction times were subtracted to account for variable motor delays in PwP. In this way, the results relate to relative changes in sensory processing rather than reflecting slower motor responses with disease progression. The Greenhouse-Geisser correction was used to adjust Fvalues and probabilities when sphericity was violated. The original degrees of freedom are presented for each analysis.

\section{Correlation analysis of disease duration}

Correlation analyses were performed on the PwP group to assess the extent to which the relative differences of reaction times for the three conditions, (auditory-visual, audiovisual-visual, audiovisualauditory), are associated with disease duration (years since symptoms onset).

\section{Miller race model}

In order to quantitatively assess the degree to which multisensory integration contributes to response times for the audiovisual condition, the Miller race model was employed [42]. Faster reaction times to the multisensory stimuli could be the result of participants responding to whichever stimulus is processed fastest, even in the absence of any interaction between the 
individual sensory stimuli. In this way, sensory processing could be considered a race between two modalities (auditory and visual in this case) on a trialby-trial basis. The race model proposed by Miller is a commonly used behavioral index of multisensory integration which takes this effect into account [36-41]. According to Miller's race model, reaction times are still expected to be faster in the multisensory condition compared with the unisensory state. This is because there are now two inputs, which can trigger a response, as opposed to just one. Whichever input is fastest, triggers a response, making a faster response more likely in the multisensory condition than if only a single stimulus was present. Miller's race model defines an upper limit for multisensory responses in this simple linear model based on the sum of the cumulative probabilities of each unisensory stimulus triggering a response. If the recorded multisensory reaction time is faster than this upper limit then violation of the race model has occurred and it must be assumed that the unisensory inputs interacted during processing (i.e. multisensory integration occurred). Failure to violate the race model, however, does not prove that the unisensory inputs did not integrate, but implies that the recorded multisensory reaction time could be explained by simple summation of unisensory probabilities. To control for false positives resulting from the multiple comparisons, $p$-values were corrected using the false discovery rate (FDR). The FDR is a sequential Bonferroni-type procedure.

\section{RESULTS}

\section{Demographics}

The demographic and neurocognitive data for the PD cohort (divided by FOG status) is given in Table 1. The 17 healthy control participants (10 Male) had a mean age of $66+/-9.7$ years (range 52-80).

\section{Hit rate analysis}

Hit rates (proportion of stimuli responded to) were consistently high across all groups (Table 2). No significant hit rate differences were found between first and last blocks of trials for any group.

\section{Reaction time}

PwP were significantly slower than controls for all conditions. Table 3 and Fig. 1 show the mean reaction times and standard deviations for each condition
Table 1

Patient Demographics by FOG status. Means shown with standard deviation in parentheses (unless median stated)

\begin{tabular}{lccc}
\hline & All PD & Freezers & Non-Freezers \\
\hline $\mathrm{N}$ & 39 & 23 & 16 \\
Age & $67.4(9.8)$ & $68.7(9.7)$ & $66.7(10.05)$ \\
Gender (M:F) & $23: 16$ & $15: 8$ & $8: 8$ \\
H\&Y stage (median) & $2.5(0.7)$ & $3.0(0.6)$ & $2.5(0.3)$ \\
Disease Duration (years) & $10.1(9.4)$ & $14.0(10.5)$ & $5.2(4.6)$ \\
UPDRS & $34.1(14)$ & $38(13)$ & $30(14)$ \\
MOCA & $24.7(4.8)$ & $24.4(3.3)$ & $26.3(3.6)$ \\
FAB & $15.7(3.3)$ & $15.4(2.8)$ & $17.1(1.5)$ \\
\hline
\end{tabular}

*indicates statistically significant difference between groups. H\&Y stage $=$ Modified Hoehn \& Yahr stage; UPDRS III = Unified Parkinson's Disease Rating Scale III total; MOCA = Montreal Cognitive Assessment total; FAB $=$ Frontal Assessment Battery total; $\mathrm{PD}=$ Parkinson's disease .

Table 2

Mean hit rate and standard deviation for control group and people with Parkinson's disease (PwP) group

\begin{tabular}{lccc}
\hline Group & A & V & AV \\
\hline PwP $(N=39)$ & $0.94(0.08)$ & $0.92(0.09)$ & $0.97(0.03)$ \\
Controls $(N=17)$ & $0.98(0.05)$ & $0.94(0.06)$ & $0.98(0.02)$ \\
\hline
\end{tabular}

$\mathrm{A}=$ auditory, $V=$ visual, $\mathrm{AV}=$ audiovisual.

Table 3

Mean and standard deviation of reaction times for control group and people with Parkinson's disease (PwP) group

\begin{tabular}{lccc}
\hline Group & A & V & AV \\
\hline PwP $(N=39)$ & $374.1(74.0)$ & $403.8(67.6)$ & $325.2(68.0)$ \\
Controls $(N=17)$ & $295.2(47.9)$ & $315.1(36.9)$ & $245.1(29.7)$ \\
\hline A = auditory-alone,$V=$ visual-alone, & AV $=$ audiovisual
\end{tabular}

(auditory-alone, visual-alone, audiovisual) and group (PwP and control participants). The mixed repeated ANOVA revealed a significant difference between the conditions' reaction times $\left(\mathrm{F}_{2,108}=84.32, P<0.001\right)$ with the fastest reaction times for the audiovisual condition. The analysis revealed significant difference between groups $\left(\mathrm{F}_{1,53}=24.1, P<0.001\right)$ with faster reaction times for all stimulus conditions in the control participants than in the participants with PD.

To investigate the significant effect of condition (auditory, visual, audiovisual), the data were submitted to a follow-up within-group between-stimulus conditions analysis. The paired $t$-tests revealed that the reaction times in the audiovisual condition (AV) were significantly faster than the reaction times for the auditory-alone (A) and visual-alone (V) conditions in the control group (auditory-alone vs audiovisual $p<0.001$; visual-alone vs audiovisual $p<0.001$ ) and the PD group (auditory-alone vs audiovisual $p<0.001$; visual-alone vs audiovisual $p<0.001)$. The analysis in 


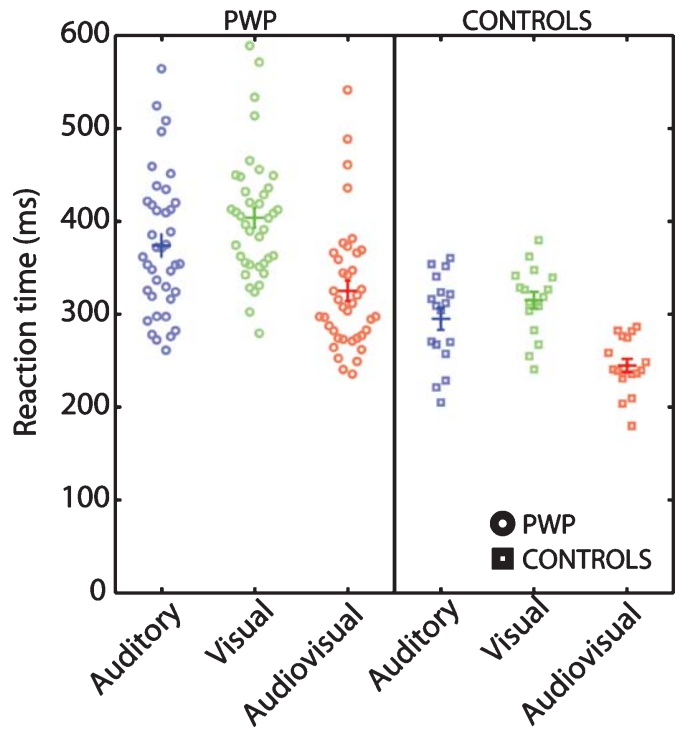

Fig. 1. Reaction times for the audio (blue), visual (green) and Audiovisual (red) conditions for both the people with Parkinson's disease (PWP, circles) and control participants (squares). The horizontal line and errorbars depict the mean and standard error of the mean.

the patients with PD revealed significant differences between the unisensory conditions; auditory-alone vs visual-alone $(p<0.001)$, while in the control participants there was no significant difference between the unisensory auditory-alone and visual-alone conditions $(p=0.26)$.

\section{FOG status and disease duration analysis}

To investigate the relationship between relative sensory processing (controlling for motor delays) and FOG status, the PD group was subdivided by
Question One of the New Freezing of Gait Questionnaire [35], as described above (Table 1). A mixed repeated ANOVA was performed with the withinparticipant factor of relative reaction time $(\mathrm{A}-\mathrm{V}$, $\mathrm{A}-\mathrm{AV}$ vs $\mathrm{A}-\mathrm{AV}$ ) and between-participant factor of FOG status (freezers vs non-freezers). The reaction times were subtracted to account for variable motor delays in PwP, which allows for the analysis of relative sensory reaction times, taking into account variable motor delays seen in PwP. In this way, the results reflect true changes in sensory processing rather than slower motor responses in freezers. Of note, no significant reaction time differences were found between first and last trial blocks for either group. The analysis revealed a significant difference between the relative reaction times $\left(F_{2,74}=67.663\right.$, $P<0.001)$. There was a significant interaction of FOG status and relative reaction time $\left(\mathrm{F}_{2,74}=3.37\right.$, $P<0.05)$. The analysis revealed no significant difference between groups across relative reaction times $\left(\mathrm{F}_{1,37}=2.39, P=0.131\right)$. The interaction effect was driven by a statistical difference $\left(\mathrm{t}_{37}=2.037, p<0.05\right)$ of the relative difference between the auditory and visual unisensory reaction times (i.e. A-V) in the freezers $(\mathrm{M}=-43.3, \mathrm{SD}=55.13 \mathrm{~ms})$ compared with non-freezers $(M=-10.32, S D=40.23 \mathrm{~ms})$. As FOG tends to occur late in the course of the idiopathic PD, efforts were made to address this strong relationship inherent in FOG studies. A follow-up Kruskal-Wallis test of disease duration (years since symptom onset) between the freezers and non-freezers was performed which revealed a statistical difference between the groups $(\mathrm{H}(1)=11.84, p<0.001)$.

This significant difference in disease duration with respect to FOG status prompted the exploration of the
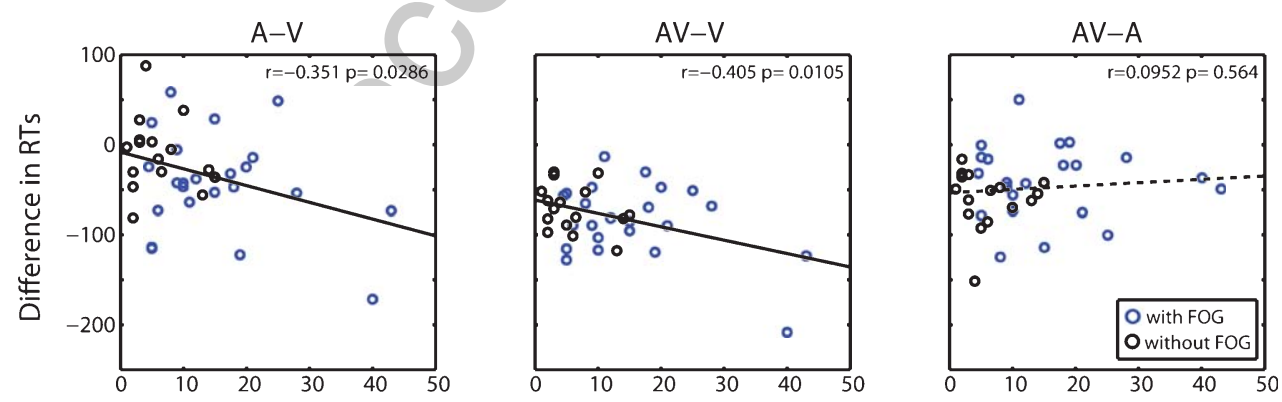

Years Since Symptom Onset (yrs)

Fig. 2. Correlation of disease duration and relative sensory processing. Scatterplots displaying on the x-axis years since symptom onset and on the $y$-axis of the left panel, the subtraction of visual from auditory reaction times (RTs); middle panel, the subtraction of visual from audiovisual reaction times; and right panel, the subtraction of auditory from audiovisual reaction times. Each circle represents a person with Parkinson's disease (with freezers indicated in blue and non-freezers indicated in black), r-values and $p$-values are shown for significant (solid lines) and non-significant (dashed lines) regression analyses. $A=$ auditory-alone, $V=$ visual-alone, $\mathrm{AV}=$ audiovisual. 
relationship between relative sensory processing (controlling for motor output delays) and disease duration, three post-hoc correlation analyses were performed on the PD group (Fig. 2). Correlation analyses were performed between years since symptom onset ( $\mathrm{x}$ axis) versus 1) auditory-alone reaction times minus visual-alone reaction times $(\mathrm{A}-\mathrm{V}) ; 2$ ) audiovisual reaction times minus visual-alone reaction times $(\mathrm{AV}-\mathrm{V})$; and 3) audiovisual reaction times minus auditory-alone reaction times (AV-A). Again, the reaction times were subtracted to account for variable motor speed in PwP. Thus any differences are due to true sensory processing differences rather than slower motor responses with disease progression.

The correlation between the subtraction of mean reaction time of auditory from visual $(\mathrm{A}-\mathrm{V})$ conditions and years since symptom onset revealed a significant relationship $\left(\mathrm{r}_{37}=-0.351, P<0.05\right)$. A similar significant relationship was found between the subtraction of mean reaction time of audiovisual from visual $(\mathrm{AV}-\mathrm{V})$ conditions and years since symptom onset $\left(\mathrm{r}_{37}=-0.415, P<0.0125\right)$. In contrast, there was no significant correlation between the subtraction of mean reaction time of auditory and visual $(\mathrm{A}-\mathrm{V})$ conditions and years since symptom onset $\left(\mathrm{r}_{37}=0.0952\right.$, $P=0.56$ ). The analysis suggests that relative delays

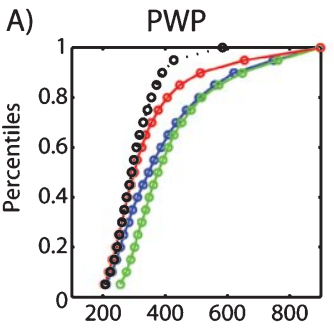

B) CONTROLS

C)

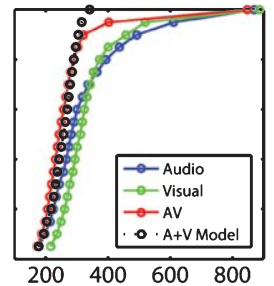

D)

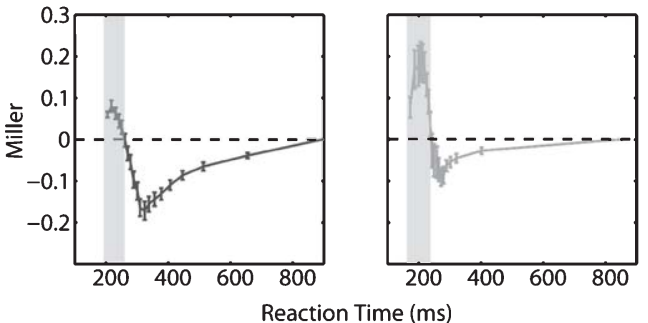

in visual processing correlate with disease duration. A follow-up ANOVA with the within-participant factor of relative reaction time (A-V, A-AV vs $\mathrm{A}-\mathrm{AV})$ and between-participant factor of FOG status (freezers vs non-freezers) resulted no significant interaction of FOG status and relative reaction times $\left(\mathrm{F}_{2,74}=0.931\right.$, $P=0.195)$. This further highlights the intricate link between FOG status and disease duration and further work is required to separate these effects.

\section{Miller Inequality}

To test the Miller race model, reaction time range was calculated across the three stimulus types for each participant. Reaction times were sorted from fastest to slowest and the reaction time distribution was then divided into quantiles from the 5th to the 100th percentile in increments of 5\% (e.g. as shown in Fig. 3A and Fig. 3B). At the individual level, a participant was said to have shown race model violation if the cumulative probability of their reaction times to the audiovisual stimulus was larger than that predicted by the race model at any quantile. We expect violations to occur in the quantiles which contain the fastest reaction times since, the faster the multisensory response, the more likely it is that multisensory

E)

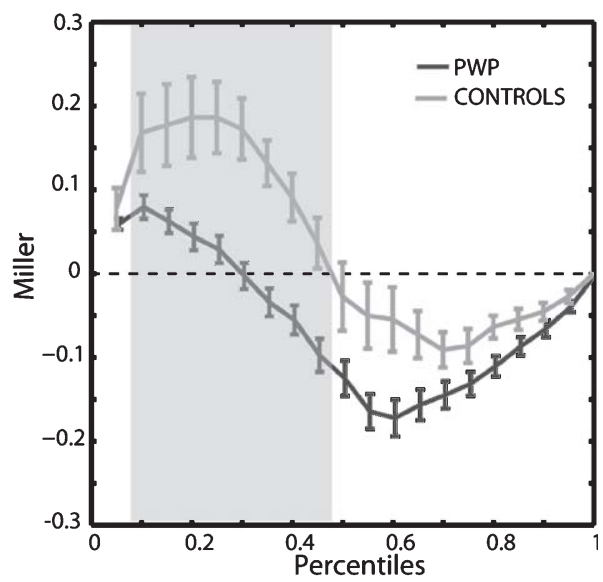

Fig. 3. A) \& B) Cumulative Probability distributions for the auditory-alone (blue), visual-alone (green), audio-visual (red) and the cumulative probability predicted by the race model (black dotted) as a function of reaction time for people with Parkinson's diseases (PwP) and aged matched controls, respectively. C) \& D) illustrate the subtraction of the multisensory cumulative probability and the cumulative probability predicted by the race model, known as the Miller inequality, as a function of reaction times for PwP (left) and aged matched controls (right), the errorbars depict standard error of the mean. The shaded areas indicate miller inequality values statistically greater than zero (dashed horizontal line) and signify race-model violation. E) The Miller inequality as a function of percentiles for PwP (dark grey) and aged matched controls (light grey). The shaded area indicates percentiles where the miller inequality is greater than zero (dashed horizontal line) for the control group and that are also significantly greater than PwP. 
facilitation has occurred. Conversely, the quantiles relating to slower multisensory reaction times are less likely to violate the race model. Testing of the Miller race model outlined above is also independent of variable motor responses as the multisensory response times are compared directly to the individual unisensory response times.

Figure 3A and B shows the cumulative probability for the auditory-alone (blue), visual-alone (green), audiovisual (red) and the cumulative probability predicted by Miller's race-model (black-dotted) for PwP and aged matched controls, respectively. The PD group had a broader cumulative probability distribution for all three conditions with onsets later than their aged matched controls. Figure 3C and D shows the subtraction of the value predicted by the race model from the audiovisual cumulative probability curve, known as the Miller inequality, as a function of reaction time divided into percentiles. Miller inequality values statistically greater than zero (dashed horizontal line) signify racemodel violation. To test for within-group violation of the race model, the Miller inequality values at each of the reaction times were submitted to one-tailed $t$-tests (greater than 0 , dashed line). The analysis revealed significant violation of the race model (shaded areas) for PwP (Fig. 3C) and aged-matched controls (Fig. 3D), thus both groups showed multisensory reaction time benefits. Interestingly, there was no significant difference in race model violation between freezers and non-freezers.

Figure 3E illustrates the Miller inequality as a function of percentile for the PD group (dark grey) and control group (light grey). To investigate differences in multisensory processing between $\mathrm{PwP}$ and controls, taking into account reaction time differences, the Miller inequalities at each percentile were submitted to unpaired $t$-tests. The analysis revealed significantly larger Miller inequality and a larger number of percentiles violating the race model (dashed line) in the control group (shaded area) than the PD group. Thus, the PD group has less enhanced multisensory processing compared with aged matched controls, as measured by violation of the race model.

\section{DISCUSSION}

Sensory and perceptual disturbances are prominent in PD and probably contribute to bradykinesia and gait disturbances [9-11]. Our results show delays in response times to visual, auditory and audiovisual stimuli in PwP compared with age-matched healthy controls. This is not surprising, given the prominence of bradykinesia in PD. However, by comparing auditory-alone, visual-alone and audio-visual responses, differences in relative sensory processing between PwP and controls suggest that sensory processing is inherently altered in PD. These changes correlate with both FOG status and disease duration, suggesting an effect that is specific to PD progression and providing a link between these sensory abnormalities and a motor feature of PD. Specifically, there is a significant difference between auditory and visual reaction times in PwP which is not present in agematched healthy controls. This relative difference is significantly greater in those with FOG and correlates with disease duration. Although multisensory facilitation occurs in PD, it is significantly less enhanced than in healthy controls. Reaction time tests represent a simplistic model for assessing sensorimotor and crosssensory function but it allows quantitative assessment of deficits which underpin more complex abnormalities of sensorimotor function in PD using a simple portable paradigm.

There is an extensive literature describing sensory deficits in PD, predominantly in response to a single sensory modality. Few studies have quantitatively reported on multisensory integration in PD and no study to date has investigated the interaction of auditory and visual modalities and their effect on reaction time. Our study has shown that both unisensory and multisensory processing abnormalities are present in patients with PD. We will discuss the unisensory and multisensory findings of the current study separately.

\section{Unisensory processing}

Our study showed that unisensory responses to both auditory and visual stimuli are slower than healthy controls. In the PD group (but not in controls) the responses to visual stimuli were significantly slower than in the auditory modality.

There is extensive clinical, behavioral, electrophysiological and imaging evidence, showing abnormal visual processing with PD progression at multiple levels from retina to visual cortex [43, 44]. Gait parameters of PwP deteriorate significantly in the absence of visual feedback [1] and FOG occurs most often when visual feedback is lacking (e.g. in dark environments) [14]. Retinal nerve fibre layer thickness [45], functional neuroimaging [44, 46] and visual evoked potential studies $[25,47]$ all provide evidence that visual processing deficits correlate with both disease duration and specific motor symptoms in PD, 
consistent with the findings of our study. Auditory processing deficits are less extensive in PD but auditory evoked potentials are abnormal in PD, suggesting both early and late information processing deficits [27, 48-52].

Motor responses to sensory stimuli test sensory processing, sensorimotor integration and motor performance. Existing reaction time studies which examine each modality in isolation, therefore, reflect sensorimotor effects rather than pure sensory ones. By comparing relative differences between reaction times to auditory and visual stimuli over a large number of trials, the current study examines sensory responses independent of a common motor output. Our study shows that visual reaction times were significantly slower compared with auditory reaction times in PD, although both were slower compared with controls. Moreover, the difference between auditory and visual response times was correlated with FOG and disease duration. The relative differences between freezers and non-freezers appears to be due to a greater reduction in auditory reaction time (i.e. faster response) in the freezers compared with controls, rather than being driven by differences in visual reaction times. This suggests a possible adaptive response in PwP where auditory processing becomes faster relative to visual processing. This difference increases with disease duration and the development of FOG. Such an adaptive process is consistent with a recent neuroimaging study which found functional reorganization of locomotor networks in PD patients with FOG which is postulated to be a maladaptive compensatory mechanism in freezers [53].

Since FOG occurs more commonly in late stage PD, it is important to be cautious when interpreting associations involving disease duration and FOG as they are closely correlated. This confounder is present to some degree in all studies of FOG. Nevertheless, our results support a disease-specific effect, independent of motor performance, rather than a corollary of multiple other neurological deficits seen in this group.

\section{Multisensory processing}

A number of studies have implicitly examined multisensory integration in PD. Studies on interactions between proprioceptive and visual information and their effect on spatial estimation have focused on spatial orientation and inherently invoked the investigation of spatial working memory, which complicates the effect of multisensory integration in PD $[1,10,11$, 54-57]. This is the first study to explicitly examine audiovisual multisensory integration in PD and we have shown that, although multisensory facilitation occurs in PwP, it is significantly less enhanced compared with age-matched healthy controls.

Animal studies have shown that kinesthetic sensory processing deficits correlate with degree of basal ganglia dopamine loss. With minor dopamine loss (e.g. in caudate nucleus only), this deficit can be overcome by integrating with visual information [58]. This effect has similarly been seen in clinical studies in PwP [11]. It is proposed that, as striatal dopamine loss worsens, the ability to compensate using sensory information is also lost. Single-cell recordings in mouse and cat have isolated large populations of multisensory neurons in the caudate and substantia nigra (cat) and dorsomedial striatum (mouse) $[31,32]$. These suggest that the basal ganglia is a multisensory hub, crucial for integration of complex sensory stimuli from multiple modalities during execution of motor output. The striatal multisensory responses can be facilitatory or inhibitory. It is probable that a similarly large proportion of human striatal neurons have the capacity for multisensory integration, refining the response to multisensory stimuli and allowing fine motor control with complex sensory inputs. The progressive loss of striatal dopaminergic innervation affects these neurons explaining the reduced multisensory facilitation in PD. Furthermore, as progressive loss of these neurons occurs over time, the sensorimotor responses become less and less refined, eventually approaching an all-or-nothing response. In this case, certain complex sensory environments could lead to dramatic augmentation of motor output by leading to a net crude facilitatory response whereas others (e.g. doorways, noise, crowds) could cause dramatic inhibition of motor output by leading to a net crude inhibitory response, causing akinesia or freezing of gait. This is consistent with existing models of FOG, which suggest that intense sensory stimulation overloads integrated parallel processing network within the basal ganglia leading to overactivity of the output nuclei of the basal ganglia causing FOG [59-61]. Cowie et al. compared the gait of PwP and healthy controls walking through doorways and showed progressive scaling of gait parameters as PwP walked through increasingly narrow doorways [62]. As FOG frequently occurs at doorways [63], it is possible that a perceptual deficit underpins the pathophysiology of FOG $[14,64]$. We posit that these sensorimotor effects occur due to multisensory interactions between visual and nonvisual sensory inputs, rather than simple unisensory deficits. 
The most dramatic multisensory effect seen in PD is that of sensory cueing on gait [65] and, in particular, on FOG [66]. Sensory cueing (i.e. the use of a temporal or spatial stimulus to facilitate motor output) is used widely in PD as a strategy to improve gait. The fact that FOG can be strikingly relieved by the addition of rhythmical sensory stimuli provides further evidence that there are significant sensory effects in PD. Given that locomotion is a highly complex multisensory task, the improvements in gait using specific sensory stimuli are probably mediated via alterations in sensory integration with motor output [67]. It should be noted that attention is a powerful modulator of these sensory effects, in particular, sensory cueing. Indeed, attentional cues alone can reduce freezing and improve gait. Our findings that multisensory integration is less enhanced in PD patients than in healthy controls could be considered to be at odds with the observation that patients with PD get significant benefit from additional sensory information such as in rhythmical cueing. It is important to highlight that the results of the current study show that multisensory integration is reduced but present in PD. We must consider the possibility that intact but diminished multisensory integration may be beneficial, as the over-integration of multisensory information seen in older adults has been linked with falls [30]. Finally, the multisensory changes seen here do not correlate with either disease duration or FOG status. This suggests that altered multisensory processing may occur even in early PD and may be a potential biomarker for the disease. Multisensory deficits have similarly been suggested as a potential biomarker in other neurodegenerative disorders, such as Niemann Pick Type C, using a similar paradigm [36].

\section{Future directions}

Rehabilitation strategies which incorporate sensory feedback have been shown to be of benefit in PD [68-74]. Specific strategies targeting multisensory integration result in behavioral and imaging changes in healthy cohorts [75-78] providing evidence that multisensory deficits can be improved with training. Such multisensory strategies have led to improvements in balance and posture in older adults [79-82] and improvements in rehabilitation following spinal cord injury and stroke $[83,84]$. Further exploration of the role of multisensory training in PD may lead to promising therapeutic strategies for mobility, safety and FOG.

The main limitation of this study is the inability to separate the effects of disease duration and FOG status. Freezing and disease duration are intricately linked. By controlling for one, the effect of the other is lost. This could be overcome by specifically recruiting patients with early FOG or those late in their disease course without FOG. This would, however, select out biologically different subtypes of PD. This may allow a greater understanding of the sensory processes underlying FOG but this subgroup analysis is beyond the scope of the current work.

As mentioned above, multisensory integration is intricately linked with attention and it is likely that attentional effects may contribute to the results seen above. Performance on attentional tasks are correlated with FOG, in particular when performed under temporal pressure $[85,86]$. Tard et al. recently examined attention in FOG using unisensory reaction times and showed no difference between freezers and nonfreezers in simple reaction times when corrected for disease duration [87]. However, when a divided attention task was performed freezers were slower. This suggests that divided attention is impaired in FOG. Future work should focus on combining these two paradigms in order to explore the parallel effects of multisensory integration and attention.

Our multisensory findings could be explained by inequality of unisensory response times. It has been shown that equivalence of unisensory responses of individual modalities leads to optimal multisensory facilitation when those modalities are combined [88, 89]. If one modality dominates (as auditory does in the PD cohort), then there is less opportunity for multisensory facilitation. The auditory response times in this study are closely correlated with multisensory facilitation. In contrast, the healthy control group displays approximately equal responses to auditory and visual stimuli, perhaps explaining the greater multisensory integration in controls compared with the PD group. Alterations in unisensory processing in PD described above may, therefore, be contributing directly to the diminished multisensory enhancement seen here. To account for this difference, the visual and auditory stimuli could be titrated for each participant to allow equivalent unisensory response times, thus eliminating this dominance effect.

Future work should include examining the effect of dopaminergic therapy on the above findings. All patients were tested in the "on"-medication state. It would be necessary, however, to confirm that our multisensory findings are similar off medication. Future studies should also include variation of detectability of unisensory stimuli to allow for optimum multisensory gain, inclusion of other sensory modalities and more complex stimuli as well as variation of timing 
between stimuli to examine the effect of temporal window of integration. Although the discussion here is in terms of specific modalities (visual and auditory), we posit that there may be a more global effect of relative sensory differences also affecting other modalities.

\section{CONCLUSION}

PD is associated with widespread sensory deficits: peripheral and central; simple and complex; unisensory and multisensory. The precise interaction that these impairments have with gait and motor control is incompletely understood. It is, however, likely that a greater understanding of these processes will have positive implications for therapeutic targets and rehabilitation.

The current study has shown that:

1. Both unisensory and multisensory delayed reaction times exist in patients with PD, in line with previous findings.

2. Relative differences in auditory and visual processing occur in PwP and correlate with FOG and longer disease duration.

3. Multisensory integration of auditory and visual stimuli is significantly less enhanced compared with age-matched healthy controls, adding to the literature supporting both simple and higherorder sensory processing abnormalities in PD.

\section{ACKNOWLEDGMENTS}

The authors express their sincere gratitude to the patients and control participants for generously donating their time to the study.

\section{FINANCIAL DISCLOSURES AND FUNDING}

This research was supported by European Union Funded Project FP7-288914-VERVE. The authors declare no conflicts of interest or financial disclosures.

\section{REFERENCES}

[1] Martens KAE, \& Almeida QJ (2011) Dissociating between sensory and perceptual deficits in PD: More than simply a motor deficit. Mov Disord, 27, 387-392.

[2] Konczak J, Sciutti A, Avanzino L, Squeri V, Gori M, Masia L, Abbruzzese G, \& Sandini G(2012) Parkinson's disease accelerates age-related decline in haptic perception by altering somatosensory integration. Brain, 135, 3371-3379.

[3] Patel N, Jankovic J, \& Hallett M(2014) Sensory aspects of movement disorders. Lancet Neurol, 13, 100-112.
[4] Doty RL (2007) Olfaction in Parkinson's disease. Parkinsonism Relat Disord, 13, S225-S228.

[5] Armstrong RA (2011) Visual symptoms in Parkinson's disease. Parkinsons Dis, 2, 1-9.

[6] Vercruysse S, Spildooren J, Heremans E, Vandenbossche J, Levin O, Wenderoth N, Swinnen SP, Janssens L, Vandenberghe W, \& Nieuwboer A (2011) Freezing in Parkinson's disease: A spatiotemporal motor disorder beyond gait. Mov Disord, 27, 254-263.

[7] Troche J, Troche MS, Berkowitz R, Grossman M, \& Reilly J (2012) Tone discrimination as a window into acoustic perceptual deficits in Parkinson's disease. Am J Speech Lang Pathol, 21, 258.

[8] Klockgether T, Borutta M, Rapp H, Spieker S, \& Dichgans $\mathrm{J}$ (1995) A defect of kinesthesia in Parkinson's disease. Mov Disord, 10, 460-465.

[9] Abbruzzese G, \& Berardelli A (2003) Sensorimotor integration in movement disorders. Mov Disord, 18, 231-240.

[10] Demirci M, Grill S, McShane L, \& Hallett M (1997) A mismatch between kinesthetic and visual perception in Parkinson's disease. Ann Neurol, 41, 781-788.

[11] Keijsers NLW, Admiraal MA, Cools AR, Bloem BR, \& Gielen CCAM (2005) Differential progression of proprioceptive and visual information processing deficits in Parkinson's disease. Eur J Neurosci, 21, 239-248.

[12] Nutt JG, Bloem BR, Giladi N, Hallett M, Horak FB, \& Nieuwboer A (2011) Freezing of gait: Moving forward on a mysterious clinical phenomenon. Lancet Neurol, 10, 734-744.

[13] Bloem BR, Hausdorff JM, Visser JE, \& Giladi N (2004) Falls and freezing of gait in Parkinson's disease: A review of two interconnected, episodic phenomena. Mov Disord, 19, 871884.

[14] Ehgoetz Martens KA, Pieruccini-Faria F, \& Almeida QJ (2013) Could sensory mechanisms be a core factor that underlies freezing of gait in Parkinson's disease? PLoS One, 8 e62602.

[15] Paunikar VM, Shastri N, \& Baig MNH (2012) Effect of parkinson's disease on audiovisual reaction time in indian population. Int J Biol Med Res, 3, 1-5.

[16] Evarts EV, Teräväinen H, \& Calne DB (1981) Reaction time in Parkinson's disease. Brain, 104, 167-186.

[17] Pullman SL, Watts RL, Juncos JL, Chase TN, \& Sanes JN (1988) Dopaminergic effects on simple and choice reaction time performance in Parkinson's disease. Neurology, 38, 249.

[18] Jordan N, Sagar HJ, \& Cooper JA (1992) Cognitive components of reaction time in Parkinson's disease. J Neurol Neurosurg Psychiatry, 55, 658-664.

[19] Yokochi F, Nakamura R, \& Narabayashi H (1985) Reaction time of patients with Parkinson's disease, with reference to asymmetry of neurological signs. J Neurol Neurosurg Psychiatry, 48, 702-705.

[20] Cooper JA, Sagar HJ, Tidswell P, \& Jordan N (1994) Slowed central processing in simple and go/no-go reaction time tasks in Parkinson's disease. Brain, 117, 517-529.

[21] Low KA, Miller J, \& Vierck E (2002) Response slowing in Parkinson's disease: A psychophysiological analysis of premotor and motor processes. Brain, 125, 1980-1994.

[22] Bloxham CA, Dick DJ, \& Moore M (1987) Reaction times and attention in Parkinson's disease. J Neurol Neurosurg Psychiatry, 50, 1178-1183.

[23] Praamstra P, Meyer AS, Cools AR, Horstink MW, \& Stegeman DF (1996) Movement preparation in Parkinson's disease. Time course and distribution of movement-related potentials in a movement precueing task. Brain, 119, 1689-1704. 
[24] Silva Lopes MD, de Souza Melo A, \& Nóbrega AC (2014) Delayed latencies of auditory evoked potential P300 are associated with the severity of Parkinson's disease in older patients. Arq Neuropsiquiatr, 72, 296-300.

[25] Özden Sener H, Akbostancı MC, Yücesan C, Dora B, \& Selçuki D (2001) Visual evoked potentials in Parkinson's disease-correlation with clinical involvement. Clin Neurol Neurosurg, 103, 147-150.

[26] Rossini PM, Traversa R, Boccasena P, Martino G, Passarelli F, Pacifici L, Bernardi G, \& Stanzioni P (1993) Parkinson's disease and somatosensory evoked potentials: Apomorphine-induced transient potentiation of frontal components. Neurology, 43, 2495.

[27] Gawel MJ, Das P, Vincent S, \& Rose FC (1981) Visual and auditory evoked responses in patients with Parkinson's disease. J Neurol Neurosurg Psychiatry, 44, 227-232.

[28] Freiherr J, Lundström JN, Habel U, \& Reetz K (2013) Multisensory integration mechanisms during aging. Front Hum Neurosci, 7, 863.

[29] Laurienti PJ, Burdette JH, Maldjian JA, \& Wallace MT (2006) Enhanced multisensory integration in older adults. Neurobiol Aging, 27, 1155-1163.

[30] Setti A, Burke KE, Kenny RA, \& Newell FN (2011) Is inefficient multisensory processing associated with falls in older people? Exp Brain Res, 209, 375-384.

[31] Nagy A, Eördegh G, Paróczy Z, Márkus Z, \& Benedek G (2006) Multisensory integration in the basal ganglia. Eur $J$ Neurosci, 24, 917-924.

[32] Reig R, \& Silberberg G (2014) Multisensory integration in the mouse striatum. Neuron, 83, 1200-1212.

[33] Sabaté M, Llanos C, \& Rodríguez M (2008) Integration of auditory and kinesthetic information in motion: Alterations in Parkinson's disease. Neuropsychology, 22, 462-468.

[34] Hughes AJ, Daniel SE, Kilford L, \& Lees AJ (1992) Accuracy of clinical diagnosis of idiopathic Parkinson's disease: A clinico-pathological study of 100 cases. J Neurol Neurosurg Psychiatry, 55, 181-184.

[35] Nieuwboer A, Herman T, Rochester L, Ehab Emil G, \& Giladi $\mathrm{N}(2008)$ The new revised freezing of gait questionnaire, a reliable and valid instrument to measure freezing in Parkinson's disease? Parkinsonism Relat Disord, 14, S68.

[36] Andrade GN, Molholm S, Butler JS, Brandwein AB, Walkley SU, \& Foxe JJ (2014) Atypical multisensory integration in Niemann-Pick type $\mathrm{C}$ disease - towards potential biomarkers. Orphanet J Rare Dis, $9,149$.

[37] Brandwein AB, Foxe JJ, Butler JS, Frey H-P, Bates JC, Shulman LH, \& Molholm S (2015) Neurophysiological indices of atypical auditory processing and multisensory integration are associated with symptom severity in autism. J Autism Dev Disord, 45, 230-244.

[38] Brandwein AB, Foxe JJ, Butler JS, Russo NN, Altschuler TS, Gomes H, \& Molholm S (2013) The development of multisensory integration in high-functioning autism: Highdensity electrical mapping and psychophysical measures reveal impairments in the processing of audiovisual inputs. Cereb Cortex, 23, 1329-1341.

[39] Brandwein AB, Foxe JJ, Russo NN, Altschuler TS, Gomes H, \& Molholm S (2011) The development of audiovisual multisensory integration across childhood and early adolescence: A high-density electrical mapping study. Cereb Cortex, 21, 1042-1055.

[40] Mercier MR, Foxe JJ, Fiebelkorn IC, Butler JS, Schwartz TH, \& Molholm S (2013) Auditory-driven phase reset in visual cortex: Human electrocorticography reveals mechanisms of early multisensory integration. NeuroImage, 79, 19-29.
[41] Molholm S, Ritter W, Javitt DC, \& Foxe JJ (2004) Multisensory visual-auditory object recognition in humans: A high-density electrical mapping study. Cereb Cortex, 14, 452465 .

[42] Miller J (1982) Divided attention: Evidence for coactivation with redundant signals. Cogn Psychol, 14, 247-279.

[43] Archibald NK, Clarke MP, Mosimann UP, \& Burn DJ (2009) The retina in Parkinson's disease. Brain, 132, 1128-1145.

[44] Cardoso EF, Fregni F, Maia FM, Melo LM, Sato JR, Cruz AC, Bianchi ET, Fernandes DB, Monteiro MLR, Barbosa ER, \& Amaro Jr E (2010) Abnormal visual activation in Parkinson's disease patients. Mov Disord, 25, 1590-1596.

[45] Garcia-Martin E, Larrosa JM, Polo V, Satue M, Marques ML, Alarcia R, Seral M, Fuertes I, Otin S, \& Pablo LE (2014) Distribution of retinal layer atrophy in patients with Parkinson disease and association with disease severity and duration. $\mathrm{Am}$ J Ophthalmol, 157, 470-472.

[46] Zhang J, Bi W, Zhang Y, Zhu M, Zhang Y, Feng H, Wang J, Zhang Y, \& Jiang T (2015) Abnormal functional connectivity density in Parkinson's disease. Behav Brain Res, 280, 113-118.

[47] Wang L, Kuroiwa Y, \& Kamitani T (1999) Visual event-related potential changes at two different tasks in nondemented Parkinson's disease. J Neurol Sci, 164, 139-147.

[48] Wright M, Geffen G, \& Geffen L (1996) ERP measures of stimulus processing during an auditory oddball task in Parkinson's disease: Evidence for an early information processing deficit. Parkinsonism Relat Disord, 2, 13-21.

[49] Philipova D, Gatchev G, Vladova T, \& Georgiev D (1997) Event-related potentials in parkinsonian patients under auditory discrimination tasks. Int J Psychophysiol, 27, 69-78.

[50] Raudino F, Garavaglia P, Beretta S, \& Pellegrini G (1997) Auditory event-related potentials in Parkinson's disease. Electromyogr Clin Neurophysiol, 37, 409-413.

[51] Pekkonen E, Ahveninen J, Virtanen J, \& Teräväinen H (1998) Parkinson's disease selectively impairs preattentive auditory processing: An MEG study. NeuroReport, 9, 2949-2952.

[52] Celik M, Seleker F, Sucu H, \& Forta H (2000) Middle latency auditory evoked potentials in patients with parkinsonism. Parkinsonism Relat Disord, 6, 95-99.

[53] Fling BW, Cohen RG, Mancini M, Carpenter SD, Fair DA, Nutt JG, \& Horak FB (2014) Functional reorganization of the locomotor network in Parkinson patients with freezing of gait. PLoS One, 9, e100291.

[54] Klockgether T, \& Dichgans J (1994) Visual control of arm movement in Parkinson's disease. Mov Disord, 9, 48-56.

[55] Adamovich SV, Berkinblit MB, Hening W, Sage J, \& Poizner $\mathrm{H}$ (2001) The interaction of visual and proprioceptive inputs in pointing to actual and remembered targets in Parkinson's disease. Neuroscience, 104, 1027-1041.

[56] Martens KAE, Ellard CG, \& Almeida QJ (2013) Dopaminergic contributions to distance estimation in Parkinson's disease - A sensory-perceptual deficit? Neuropsychologia, 51, 14261434.

[57] Barnett-Cowan M, Dyde RT, Fox SH, Moro E, Hutchison WD, \& Harris LR (2010) Multisensory determinants of orientation perception in Parkinson's disease. Neuroscience, 167, 11381150.

[58] Cools AR, Jaspers R, Kolasiewicz W, Sontag KH, \& Wolfarth S (1983) Substantia nigra as a station that not only transmits, but also transforms, incoming signals for its behavioural expression: Striatal dopamine and GABA-mediated responses of pars reticulata neurons. Behav Brain Res, 7, 39-49.

[59] Lewis SJG, \& Barker RA (2009) A pathophysiological model of freezing of gait in Parkinson's disease. Parkinsonism Relat Disord, 15, 333-338. 
[60] Shine JM, Moustafa AA, Matar E, Frank MJ, \& Lewis SJG (2013) The role of frontostriatal impairment in freezing of gait in Parkinson's disease. Front Syst Neurosci, 7, 61.

[61] Lewis SJG, \& Shine JM (2014) The next step: A common neural mechanism for freezing of gait. Neuroscientist, doi: 10.1177/1073858414559101.

[62] Cowie D, Limousin P, Peters A, \& Day BL (2010) Insights into the neural control of locomotion from walking through doorways in Parkinson's disease. Neuropsychologia, 48, 2750-2757.

[63] Cowie D, Limousin P, Peters A, Hariz M, \& Day BL (2012) Doorway-provoked freezing of gait in Parkinson's disease. Mov Disord, 27, 492-499.

[64] Nantel J, McDonald JC, Tan S, \& Bronte-Stewart H (2012) Deficits in visuospatial processing contribute to quantitative measures of freezing of gait in Parkinson's disease. Neuroscience, 221, 151-156.

[65] Spaulding SJ, Barber B, Colby M, Cormack B, Mick T, \& Jenkins ME (2013) Cueing and gait improvement among people with Parkinson's disease: A meta-analysis. Arch Phys Med Rehab, 94, 562-570.

[66] Lee SJ, Yoo JY, Ryu JS, Park HK, \& Chung SJ (2012) The effects of visual and auditory cues on freezing of gait in patients with Parkinson disease. Am J Phys Med Rehab, 91, 2-11.

[67] Campos JL, Butler JS, \& Bülthoff HH (2012) Multisensory integration in the estimation of walked distances. Exp Brain Res, 218, 551-565.

[68] Baram Y (2013) Virtual sensory feedback for gait improvement in neurological patients. Front Neurol, 4, 138

[69] Zalecki T, Gorecka-Mazur A, Pietraszko W, Surowka AD, Novak P, Moskala M, \& Krygowska-Wajs A (2013) Visual feedback training using WII Fit improves balance in Parkinson's disease. Folia Med Cracov, 53, 65-78.

[70] Lefaivre SC, \& Almeida QJ (2015) Can sensory attention focused exercise facilitate the utilization of proprioception for improved balance control in PD? Gait Posture, 41, 630-633.

[71] Pelosin E (2014) Proprioceptive rehabilitation of upper limb dysfunction in movement disorders: A clinical perspective. Front Hum Neurosci, 8, 961.

[72] van den Heuvel MRC, van Wegen EEH, de Goede CJT, Burgers-Bots IAL, Beek PJ, Daffertshofer A, \& Kwakkel G (2013) The effects of augmented visual feedback during balance training in Parkinson's disease: Study design of a randomized clinical trial. BMC Neurol, 13, 137.

[73] Mirelman A, Herman T, Nicolai S, Zijlstra A, Zijlstra W, Becker C, Chiari L, \& Hausdorff JM (2011) AudioBiofeedback training for posture and balance in Patients with Parkinson's disease. J NeuroEngineering Rehabil, 8, 35.

[74] Sage MD, \& Almeida QJ (2010) A positive influence of vision on motor symptoms during sensory attention focused exercise for Parkinson's disease. Mov Disord, 25, 64-69.
[75] Butler AJ, James TW, \& James KH (2011) Enhanced multisensory integration and motor reactivation after active motor learning of audiovisual associations. J Cogn Neurosci, 23, 3515-3528.

[76] Powers AR, Hillock AR, \& Wallace MT (2009) Perceptual training narrows the temporal window of multisensory binding. J Neurosci, 29, 12265-12274.

[77] Setti A, Stapleton J, Leahy D, Walsh C, Kenny RA, \& Newell FN (2014) Improving the efficiency of multisensory integration in older adults: Audio-visual temporal discrimination training reduces susceptibility to the sound-induced flash illusion. Neuropsychologia, 61, 259-268.

[78] Bernstein LE, Auer ET, Eberhardt SP, \& Jiang J (2013) Auditory perceptual learning for speech perception can be enhanced by audiovisual training. Front Neurosci, 7, 34.

[79] Kristinsdottir EK, \& Baldursdottir B (2014) Effect of multisensory balance training for unsteady elderly people: Pilot study of the "Reykjavik model". Disabil Rehabil, 36, 12111218.

[80] Alfieri FM, Riberto M, Gatz LS, Ribeiro CPC, Lopes JAF, \& Battistella LR (2012) Comparison of multisensory and strength training for postural control in the elderly. Clin Interv Aging, 7, 119-125.

[81] Alfieri FM, Riberto M, Gatz LS, Ribeiro CPC, Lopes JAF, \& Battistella LR (2010) Functional mobility and balance in community-dwelling elderly submitted to multisensory versus strength exercises. Clin Interv Aging, 5, 181-185.

[82] Hu MH, \& Woollacott MH (1994) Multisensory training of standing balance in older adults: II. Kinematic and electromyographic postural responses. J Gerontol, 49, M62-M71.

[83] Yen S-C, Landry JM, \& Wu M (2014) Augmented multisensory feedback enhances locomotor adaptation in humans with incomplete spinal cord injury. Hum Mov Sci, 35, 80-93.

[84] Johansson BB (2012) Multisensory stimulation in stroke rehabilitation. Front Hum Neurosci, 6, 60.

[85] Shine JM, Naismith SL, Palavra NC, Lewis SJG, Moore ST, Dilda V, \& Morris TR (2013) Attentional set-shifting deficits correlate with the severity of freezing of gait in Parkinson's disease. Parkinsonism Relat Disord, 19, 388-390.

[86] Naismith SL, Shine JM, \& Lewis SJG (2010) The specific contributions of set-shifting to freezing of gait in Parkinson's disease. Mov Disord, 25, 1000-1004.

[87] Tard C, Delval A, Duhamel A, Moreau C, Devos D, Defebvre L, \& Dujardin K (2015) Specific attentional disorders and freezing of gait in Parkinson's disease. J Parkinsons Dis, 5, 379-387.

[88] Ernst MO, \& Banks MS (2002) Humans integrate visual and haptic information in a statistically optimal fashion. Nature, 415, 429-433.

[89] Alais D, \& Burr D (2004) The ventriloquist effect results from near-optimal bimodal integration. Curr Biol, 14, 257-262. 